\title{
Spectral and Spatial Quality Assessment of IHS and Wavelet Based Pan-sharpening Techniques for High Resolution Satellite Imagery
}

\author{
Farzaneh DadrasJavan, Farhad Samadzadegan, Fatemeh Fathollahi \\ Department of Geomatics, University College of Engineering, University of Tehran, Tehran, Iran \\ fdadrasjavan@ut.ac.ir, samadz@ut.ac.ir,fz.fathollahi@ut.ac.ir
}

\begin{abstract}
Over last decades, a wide range of pan-sharpening methods have been proposed to synthesize images in a way that contain both high spatial and spectral characteristics of the multispectral and panchromatic images in high resolution satellite imagery. Amongst all improved methodologies, two different scenarios of Wavelet based and IHS based strategies brought up to be more appealing. Until now, lots of modifications and integrations are also proposed for these methods and variety of new techniques are presented. In this paper, the potential of different IHS-based and wavelet-based pansharpening techniques is studied and evaluated. For the purpose, high resolution images of worldview2 satellite imagery are used and pan-sharpened images are generated based on 9 wavelet based and 6 IHS based methods. Achieved results clearly show the superiority of GIHS method as an optimum solution to keep both spectral and spatial characteristics if input images in fused image.
\end{abstract}

Keywords: Satellite imagery, Image Fusion, Spatial and Spectral quality, comparative study, HIS, Wavelet

\section{Introduction}

The application of satellite images is still growing due to the rapid development in sensor technologies. Technological limitations in producing images with both high spectral and spatial resolutions, led to the pan-sharpening (i.e. image fusion) process which produces synthesized multispectral image with both high spectral and spatial resolution. Considering the importance of pan-sharpening process, till now variety of pan-sharpening methods have been proposed and applied to various high resolution satellite imagery (Yuhendra et al., 2012; Asha \& Philip, 2012; Amolins et al., 2007, Zhang and Huang, 2015; Liu et al., 2017).

One of the common ideas in pan-sharpen image generation, is to extract the spatial details from the Pan image and to add them somehow to the MS bands, those suffer from lack of spatial information. Multi resolution analysis is known to be the most common tool for this purpose. Variety of image fusion procedures have been proposed using the multi resolution analysis based on the discrete wavelet transform (DWT). Wavelet transformation is employed to decompose images into different resolution levels for multi resolution analysis which can be utilized for fusing images at different resolution levels (Zhang \& Hong, 2005, Amolins et al., 2007).

On the other hand, there are also other widely used techniques which are mainly named as component substitution methods (Dou et al., 2007). The main procedure of these methods is to apply a predefined transformation to multispectral bands and then to substitute one of the obtained components by Pan 
Farzaneh Dadras avan, Farhad Samadzadegan, Fatemeh Fathollahi; Spectral and Spatial Quality Assessment of IHS and Wavelet Based Pan-sharpening Techniques for High Resolution Satellite Imagery, Advances in I mage and Video Processing, Volume 6 No 2, April (2018); pp: 1-15

image. Component substitution methods usually yield to images with high spatial qualities while the wavelet methods usually perform well spectrally ( Kim et al., 2011). Based on these differences, many researches try to combine these two procedures ( Kim et al., 2011; González-Audícana et al., 2005; Zhang \& Hong, 2005 ). The most proposed schemes combine the advantages of wavelets and IntensityHue-Saturation (IHS) or wavelets and Principal Component Analysis (PCA) based fusion methods. The idea to merge the procedures of wavelets with HIS or PCA is to improve the results spectrally by injecting the high frequency detail information of Pan to the component I/PC1 instead of replacing Pan with I or PC1 component of the spectral image ( $I$ is the intensity of multispectral image and PC1 is the first principal component of Pan image).

In this paper, a short review on wavelet and IHS based fusion methods is presented and using worldview-2 satellite imagery, their capabilities in generating pan-sharpen images with high spatial and spectral resolutions, are investigated. Moreover, both spatial and spectral quality metrics are developed to compare fusion results.

\section{Review on Pan-sharpening Techniques and Evaluation Metrics}

Despite the diversity in current pan-sharpening methods, most of them can generally be classified into Component Substitution (CS) and Multi-Resolution Analysis (MRA) methods (Zhang and Huang, 2015). The Component Substitution based techniques commonly used in literatures are: Intensity Hue Saturation, Principal Component Analysis (PCA), Arithmetic Combinations and Brovey Method. These techniques are simple and are reported to provide better spatial quality in final fused image (Kolekar and Shelkikar, 2016). Amongst these methods, colour transform based fusion methods (such as HIS) have become popular mainly because of their simplicity, fast computational process and also ability to preserve spatial characteristic of the Pan image (Tu et al., 2004; Choi, 2006). On the other hand, MRA techniques involve transformation of source images into different scales before applying fusion rules. The commonly used MRA techniques are pyramid, wavelet, Contourlet and Curvelet transforms (Kolekar and Shelkikar, 2016). A popular multi resolution transform is the Discrete Wavelet Transform (DWT) hich is used for pixel-level image fusion algorithms (Kolekar and Shelkikar, 2016).

\subsection{Wavelet-Based Pan-Sharpening Techniques}

Numerous fusion methods are developed based on the discrete wavelet transform (DWT) those are usually different in the algorithm of image decomposition or in the way of detail information injection, i.e. substitution, addition or weighted models (Amolins et al., 2007). The Undecimated Discrete Wavelet, known as "a' trous" (meaning "with holes"), yields to shift-invariant discrete wavelet decomposition which is required in the application of image fusion Kim et. al., 2011; Mitchell 2010; Amolins et. al., 2007).

The process of pan-sharpening based on wavelets, consists of three main stages. Firstly, the input images should be transformed to the wavelet domain. After transformation, detail coefficients (related to high frequency information of the image in different scales) and an approximation image (related to low frequency information of the image at the last scale) will be obtained. Then in the second step, the detail and approximation coefficients of the decomposed images are combined to synthesize the fused decomposition coefficients. The third step is to reconstruct the final fused image using the inverse wavelet transformation (Mitchell, 2010; Amolins et al., 2007, Kolekar and Shelkikar, 2016). In each step, some important decisions such as the scheme of wavelet decomposition and the method to combine the coefficients should be made before the fusion process. 


\subsubsection{Decomposition Stage}

There are several different methods to apply the wavelet transformation or in the other word to decompose an image into wavelet coefficients. The Mallat's algorithm uses an orthonormal basis with decimated approach, but the transformation is not shift-invariant, which can be a problem in data fusion. Instead, "a` trous" scheme which is an undecimated discrete wavelet transformation, yields to shift-invariant discrete wavelet decomposition (Amolins et al., 2007; González-Audícana et al., 2005; Rockinger, 1996). In this paper the undecimated wavelet which is a non-orthogonal, redundant transformation is applied.

\subsubsection{Methods to Combine Different Coefficients}

The important stage in the wavelet-based fusion process is how to combine the coefficients of the decomposed Pan and MS images or how to inject the detail information to MS bands. The most common methods are substitution, addition or weighted models (Amolins et al., 2007).

- Substitution. In substitution schemes, after applying wavelet transformation, the detail coefficients (or wavelet planes) of one image are substituted by the detail coefficients of the other image coefficients (Amolins et al., 2007; Kim, et. al., 2011).

- Addition. When applying undecimated wavelet transformation, the input images have the same size as the wavelet planes. So one of the input images can be directly added to the wavelet planes obtained after applying undecimated wavelet transformation to the second image (Amolins et al., 2007).

- Weighted Models. In IHS method, a weighted model is used to combine either the approximation or the detail coefficients obtained after applying wavelet transformation to both input images (González-Audícana et al., 2004).

\subsubsection{Different Pan-sharpening Schemes}

Most well-known wavelet-based pan-sharpening methods with the 'a' trous' decomposition scheme can be summarized as follows:

- Substitutive Wavelet (SW), (Amolins et al., 2007; Kim et al., 2011).

- Additive Wavelet (AW) (Amolins et al., 2007).

- Substitute Wavelet Intensity (SWI) (González-Audícana et al., 2004).

- Additive Wavelet Intensity (AWI) (Nunez et al., 1999).

- Additive Wavelet Luminance Proportional (AWLP) ( Otazu et al., 2005; Kim et al., 2011).

- Weighted Wavelet Intensity (WWI) (Zhang \& Hong, 2005).

- Substitute Wavelet Principal Component (SWPC) (González-Audícana et al., 2004).

- Additive Wavelet Principal Component (AWPC) (González-Audícana et al., 2005).

In Table 1 at jth level, $W_{P a n_{j}}$ is the wavelet plane of the Pan. I in Table 1 refers to Intensity component obtained after the IHS transformation while PC1 refers to first principal component obtained after principal component transformation. Also LL refers to the approximation image obtained at the last level of wavelet decomposition, P refers to Pan and I refers to Intensity image and w1 and w2 are calculated according to equation 1.

$$
\begin{aligned}
& L L^{\prime}=w 1 \times L L^{P}+w 2 \times L L^{I} \\
& w 1=\operatorname{corr}\left(L L^{P}, L L^{I}\right), \quad w 1+w 2=1
\end{aligned}
$$


Farzaneh Dadrasjavan, Farhad Samadzadegan, Fatemeh Fathollahi; Spectral and Spatial Quality Assessment of IHS and Wavelet Based Pan-sharpening Techniques for High Resolution Satellite I magery, Advances in Image and Video Processing, Volume 6 No 2, April (2018); pp: 1-15

\subsection{IHS-Based Pan-Sharpening Techniques}

The IHS method has a standard procedure while its major limitation is in keeping spectral content of the MS bands, which is famous as color/spectral distortion. Also it has limitation of using only three bands at once. It has been proved that the main reason for spectral distortion is the difference between the spectral response of the Pan image and intensity component of MS image (Tu et al., 2001). Different solutions have been proposed to overcome spectral distortion in color transform based methods by minimizing the Pan and Intensity difference and also to modify the method in order to be capable of using an arbitrary number of spectral bands (Tu et al., 2007; Choi, 2006, Aiazzi and Baronti, 2007, Xu et al., 2008).

These techniques can be divided into three main groups. First group, taking into account the spectral response plot of the sensor, have extended traditional three dimensional IHS to use all the bands whose spectral range falls within the range of the Pan image (Tu et al., 2004). To improve IHS extension, some coefficients have been proposed for each MS bands just based on try and error (Tu et al., 2004; Choi, 2006). Second group researches have tried to determine the quota of each MS band using the area under the spectral response functions (González-Audícana et al., 2006) and the third group have applied the mathematical methods to approximate Pan by means of the available MS bands (Aiazzi et al., 2007; Xu et al., 2008; Rahmani et al., 2010).

In IHS based fusion methods, after applying IHS transformation on the image, intensity (I), hue (H) and saturation (S) will be obtained with the I component to appear like Pan image (Zhang \& Hong, 2005). Using IHS characteristic, pan-sharpened MS bands can be achieved through the following steps (Tu et al., 2001; Zhang \& Hong, 2005):

a) Apply IHS transformation to RGB set of resampled MS image.

b) Perform histogram matching between Pan and I.

c) Replace I component by modified Pan image.

d) Apply inverse IHS transformation to data

e) The simplified and more efficient form of the above stages which avoids numerous multiplications is as follows (Tu et al., 2004):

$$
F_{i}=M S_{i}+\delta
$$

Where $\delta=$ Pan $-\mathrm{I}$ and $\mathrm{F}_{\mathrm{i}}$ and $M S_{i}$ refer to the ith band of the fused and multispectral images respectively. Tu et al. (2001) proved that the reason of the color distortion is the change of $\delta$ component during fusion process. He also demonstrated that the difference between I and Pan leads to the change of $\delta$. To IHS problem, the literature shows some suggestions in which the main effort is to modify I to be more similar to Pan image. IHS methods mostly applied for fusion are summarized as follow Equations are presented in Table 2):

- Generalized IHS (GIHS) (Tu et al., 2004, 2007): considering the relative spectral response of the IKONOS and the fact that the spectral range of the Pan covers also Near Infra-Red (NIR) band besides R, G and B bands have extended the traditional three-order transformation to four-order one.

- Generalized IHS with Tradeoff Parameter (GIHS-TP), Choi, (2006) has proposed the minimization problem for the IHS method and introduced $I_{\text {new }}$.

- GIHS with Best Tradeoff Parameter (GIHS-BTP), Tu et al., (2007) has modified Choi method using a simple energy-normalization procedure. Tu et al. demonstrated that their approach results to better preservation of spatial information than that of Choi's method. 
- GIHS with Adaptive Weights (GIHS-AW), Aiazzi and Baronti (2007) have proposed to perform a linear regression between Pan and MS bands. In the proposed scheme, a synthetic intensity, having a minimum mean square error (MSE) with respect to the reduced Pan (i.e. at the spatial scale of the original MS image), is computed. The intensity I have been assumed as a linear combination of MS bands (equation 6) with unknown coefficients $\left(w_{i}\right)$ and a bias (b) which are computed using a linear regression algorithm (Aiazzi et al., 2007).

- Improved GIHS with Adaptive Weights (IGIHS-AW), the method can extend traditional three-order transformations to an arbitrary order and all the MS bands could be fused at the same time (Xu et al., 2008).

\subsection{Spectral Quality Assessment Metrics}

Spectral image fusion quality assessment metrics are classified based on the level of spectral information, considered in quality assessment process (Alparone et al., 2006; Thomas and Wald, 2006). Traditionally, these metrics are classified as mono-modal and multi-modal techniques (Xydeas and Petrovic, 2000). A mono-modal metric considers a single image layer while a multi-modal metric considers several image channels.

Mono modals: Correlation Coefficients (CC) between the original and the fused images; Universal Quality Index (UQI) which measures the salient information contained in reference image which has been transformed into the fused image (Thomas and Wald, 2006);

Multi Modals: Spectral Angle Mapper (SAM) that obtains the angles formed ( $\alpha$ ) between the reference spectrum and the fused image spectrum (Leung et al., 2001); The ERGAS index or Relative Adimensional Global Error in the fusion (Wald, 2000; Riahi et al., 2009); and Spectral Correlation Mapper (SCM) (Van der meer, 2006).

The quality assessment metrics are presented in Table 3 where $\mathrm{x}$ and $\mathrm{y}$ stand for fused and reference images, $\bar{x}$ and $\bar{y}$ are the local sample means of $x$ and $y, \sigma x$ and $\sigma y$ are the local sample standard deviations of $x$ and $y$, and $\sigma x y$ is the sample cross correlation of $x$ and $y$ after removing their means. $S$ is the number of spectral bands of images or the dimension of the spectral space, $A=(A 1, A 2, A 3, \ldots, A S)$ and $B=(B 1, B 2, B 3, \ldots, B S)$ are two spectral vectors with same wavelength from the reference and fused images respectively. $\mathrm{Mi}$ is the mean value for the original images, $\mathrm{Bi}$ represents bands of images and GSDPan and GSDMS stand for the ground sampling resolution of initial panchromatic and multi spectral images, respectively

\subsection{Spatial Quality Assessment Metrics}

In addition to preserving the spectral information of the input MS image, a fused image should contain the spatial information of the Pan image, as well, especially in high resolution satellite imagery which are usually used for urban area mapping (Javan et al., 2013). The spatial metrics usually are used for pan-sharpening quality evaluation are presented as CC, UQI, spatial ERGAS (ERGASs) proposed by LilloSaavedra et al. (2005) and spatial CC (sCC) proposed by Zhoue et al. (1998).

\section{Experiments and Results}

To evaluate and compare the capabilities of discussed image fusion methods, all are applied for Pansharpening of WV2 satellite imagery. The evaluation strategy is presented in Figure 1. As it's depicted, in the first step, initial Pan and MS images are down sampled and Low resolution Pan (L Pan) and multi spectral (L MS) images are generated. Using this new images, Pansharpen image (Fused L image) is generated based on Wald strategy (). Then quality assessment is applied comparing the generated fused image with initial multi spectral image (H MS) spectrally and with initial Pan image (H Pan) spatially. 
Farzaneh Dadrasjavan, Farhad Samadzadegan, Fatemeh Fathollahi; Spectral and Spatial Quality Assessment of IHS and Wavelet Based Pan-sharpening Techniques for High Resolution Satellite I magery, Advances in Image and Video Processing, Volume 6 No 2, April (2018); pp: 1-15

\subsection{Data Set}

The WV2 data set which is from an urban area of Melbourne city (Figure 2). WV2 has 8 spectral bands of $500 \times 500$ pixels and a Pan band of $2000 \times 2000$ pixels with $2 \mathrm{~m}$ and $0.5 \mathrm{~m}$ resolutions, respectively. The MS bands are up-sampled and the pairs of images were geometrically registered to each other. $\mathrm{A}$ part of images ( $500 \times 500$ pixels) is extracted to compare the visual effects of different fused images.

\subsection{Wavelet Based Image Fusion Results}

In IHS experiment, the undecimated transformation scheme was used to decompose the input images in all the methods and the level of decomposition was set to 2 for all the resultant images. To compare the visual effect of the different fused images, a part of the resultant images ( $500 \times 500$ pixels) is extracted and shown in Figure 3. From Figure 3, it can be seen that the spatial resolutions of all the resultant images are improved through the fusion process in comparison with the original MS. Considering the resultant images of additive schemes, it seems that they suffer from some distortions along the lines in the images exactly similar to those that can be seen along the edges of the original MS. The reason is that in additive schemes, the low resolution image (MS itself or Intensity component or PC1) is directly added to the detail coefficients of the Pan without being decomposed.

\subsection{IHS based Image Fusion Results}

Considering the spectral response function of the WV2 sensors (depicted in Figure 4) IHS-based methods for WV2 can profit from the additional yellow and red edge bands whose spectral range fall within the range of the Pan (Figure4). So, in the modified scenario for WV2, the five bands of blue, green, yellow, red and red edge were used in the process to improve the spectral quality of the fused image. In IHS experiment all the mentioned methods reviewed in chapter 2, were applied to WV2 data. To compare the effects of the fusion process visually, a part of resultant images ( $500 \times 500$ pixels) is extracted and shown in Figure 5. From results, although visual comparison of images is not convenient, but it is clearly observed that all methods improved the spatial quality of MS image. But some of the methods like GIHSAW and IHS resulted in better spatial quality while IHS presented spectral distortion.

\subsection{Spectral Quality Assessment}

To evaluate the spectral quality of the fused images, the Pan and original MS images were degraded to a resolution of 2 and $4 \mathrm{~m}$ respectively and were fused at a $4 \mathrm{~m}$ resolution. The spectral quality was evaluated by comparing the spectral information of fused image to that of the original MS image applying spectral quality assessment metrics. Table 4 and 5 show the results of spectral metrics calculated to evaluate the fused images.

To provide the capability of visual comparison of results, they are also depicted in Figure 6. To avoid messy figure, results are presented for the mean value of $R, G$ and $B$ bands. Comparing the values in Table 4 and Figure 6, it can be concluded that the method SWPC, while averaging all its bands, yields to a fused image with superior spectral quality. However, it can be seen that there is no significant difference between results of SWPC and SWI methods, which shows the success of these hybrid methods in preservation of spectral information. It is also observed that substitution schemes usually result to better preservation of spectral content of the original MS image in comparison with additive schemes (see Figures 6.b and 6.c).

From Table 5, it can be concluded that all mentioned IHS based methods have better spectral quality in comparison with traditional IHS method and the superior spectral quality was obtained by IGIHS-AW 
method. As IHS method is designed to minimize spectral distortion by keeping the spectral vector of the fused image parallel to that of the original MS, the obtained value for SAM is near to zero.

\subsection{Spatial Quality Assessment}

Figure 7 presents the results of pan-sharpening methods based on spatial metrics while comparing generated pan-sharpen and Pan images. The values of ERGASs, UQI and SCC calculated for three spectral bands ( $R, G$ and $B$ ) are averaged and demonstrated in Figure 7.

From Tables 6 and 7 also Figure 7, all calculated metrics, address the WWI method as the superior method in terms of spatial quality while the SCC metric has addressed the SW method. It shows that the idea to keep the Pan approximation partially, instead of ignoring it all, can improve the spatial quality of the fused image. Comparing the additive schemes with substitutive ones, it can be found that the metrics SCC and UQI show better spatial qualities by the results of additive scheme, while the metrics SCC and ERGASs, show the better functionality of the substitutive schemes in keeping the spatial information. It seems that the metrics SCC and ERGASs are more sensitive to those distortion effects of additive schemes along the lines that can be easily observed in the results.

\section{Discussions}

6 common IHS based fusion methods and 9 different wavelet based fusion methods are evaluated based on spectral and spatial quality assessment metrics. Results clearly show the total spatial dominance of IHS based methods and spectral superiority of Wavelet based Methods. Results also revealed that in wavelet based method SWPC and WWI show the best results for spectral and spatial quality respectively. Moreover, among IHS based methods, IGIHS-AW and traditional IHS methods present the best for spectral and spatial quality respectively.

On the other hand, to have better sense over the results which are discussed in previous section, we applied an averaging strategy of evaluation and comparison. For the purpose, the values for each metric are normalized based on their global minimum and maximum values in each table of 4, 5, 6 and 7 . Then, the normalized values are averaged for each method in both case of spectral and spatial assessment as a grade. Results are presented in table 8 and 9 . The best quality results are marked in both spectral and spatial case. Moreover, as in lots of applications both spectral and spatial qualities are important, users should apply methods fairly enjoy both spectral and spatial characteristics. So, the grades in each case are again normalized with respect to their $\mathrm{min} / \mathrm{max}$ values and averaged and the final score for each of these wavelet based and IHS methods are computed.

As it is presented in tables 8 and 9, while considering spectral quality, GIHS and for spatial quality, IHS presents the best results. Moreover if both spectral and spatial qualities are important, GIHS method can perform better. Although it is not the best spectrally or spatially, it can somehow present both in an acceptable level.

\section{Conclusion}

The widespread use of pan-sharpening in generation of high spatial and spectral quality in high resolution satellite imagery, has led to a wide range of image fusion techniques. In this paper the potential of wavelet based and IHS based techniques as most common Pan-sharpening methods have been inspected. 6 IHS based and 9 Wavelet based methods were developed and their capabilities in generating fused images were studied based on Worldview-2 satellite imagery. Obtained results clearly showed that if spectral resolution is considered, SWPC and GIHS are the most wisdom selection. On the other hand, in case of spatial resolution the classic IHS and WWI methods are the best. Moreover, as both spectral and spatial quality are important in lots of application, results showed that GIHS and 
Farzaneh Dadrasjavan, Farhad Samadzadegan, Fatemeh Fathollahi; Spectral and Spatial Quality Assessment of IHS and Wavelet Based Pan-sharpening Techniques for High Resolution Satellite I magery, Advances in Image and Video Processing, Volume 6 No 2, April (2018); pp: 1-15

SW is one of the best which showed acceptable results in both cases and can be applied for lots of Pansharpening applications.

\section{REFERENCES}

[1] Aiazzi, B., S. Baronti, and M. Selva. "Improving Component Substitution Pansharpening Through Multivariate Regression of MS+ Pan Data." IEEE Transactions on Geoscience and Remote Sensing 45 (2007): 3230-3239.

[2] Alimuddin, I., Sumantyo, J. T. S., \& Kuze, H. (2012). Assessment of pan-sharpening methods applied to image fusion of remotely sensed multi-band data. International Journal of Applied Earth Observation and Geoinformation, 18, 165-175.

[3] Amolins, K., Zhang, Y., \& Dare, P. (2007). Wavelet based image fusion techniques-An introduction, review and comparison. ISPRS Journal of Photogrammetry and Remote Sensing, 62(4), 249-263.

[4] Asha, G., and Annes Philip. "A review on pixel level satellite image fusion." Int. J. Comput. Appl 1 (2012): 15-19.

[5] Choi, M. (2006). A new intensity-hue-saturation fusion approach to image fusion with a tradeoff parameter. IEEE Transactions on Geoscience and Remote sensing, 44(6), 1672-1682.

[6] Javan, F. D., Samadzadegan, F., \& Reinartz, P. (2013). Spatial quality assessment of pan-sharpened high resolution satellite imagery based on an automatically estimated edge based metric. Remote Sensing, 5(12), 6539-6559.

[7] Dou, W., Chen, Y., Li, X., \& Sui, D. Z. (2007). A general framework for component substitution image fusion: An implementation using the fast image fusion method. Computers \& Geosciences, 33(2), 219228.

[8] González-Audícana, M., Otazu, X., Fors, O., \& Alvarez-Mozos, J. (2006). A low computational-cost method to fuse IKONOS images using the spectral response function of its sensors. IEEE Transactions on Geoscience and Remote Sensing, 44(6), 1683-1691.

[9] Kim, Y., Lee, C., Han, D., Kim, Y., \& Kim, Y. (2011). Improved additive-wavelet image fusion. IEEE Geoscience and Remote Sensing Letters, 8(2), 263-267.

[10] Kolekar, M. N. B., \& Shelkikar, R. P. A . (2016). A Review on Wavelet transform based image fusion and classification. International Journal of Application or Innovation in Engineering \& Management (IJAIEM), 5 (3), 111-115

[11] Leung, L. W., King, B., \& Vohora, V. (2001, November). Comparison of image data fusion techniques using entropy and INI. In Paper presented at the 22nd Asian Conference on Remote Sensing (Vol. 5, p. 9).

[12] Liu, X., Wang, Y., \& Liu, Q. (2017). Remote Sensing Image Fusion Based on Two-stream Fusion Network. arXiv preprint arXiv:1711.02549.

[13] Lillo-Saavedra, M., \& Gonzalo, C. (2006). Spectral or spatial quality for fused satellite imagery? A tradeoff solution using the wavelet à trous algorithm. International Journal of Remote Sensing, 27(7), 14531464. 
[14] Mallat, S. G. (1989). A theory for multiresolution signal decomposition: the wavelet representation. IEEE transactions on pattern analysis and machine intelligence, 11(7), 674-693.

[15] Mitchell, H. B. (2010). Image fusion: theories, techniques and applications. Springer Science \& Business Media.

[16] Nunez, J., Otazu, X., Fors, O., Prades, A., Pala, V., \& Arbiol, R. (1999). Multiresolution-based image fusion with additive wavelet decomposition. IEEE Transactions on Geoscience and Remote sensing, 37(3), 1204-1211.

[17] Rahmani, S., Strait, M., Merkurjev, D., Moeller, M., \& Wittman, T. (2010). An adaptive IHS pansharpening method. IEEE Geoscience and Remote Sensing Letters, 7(4), 746-750.

[18] Riyahi, R., Kleinn, C., \& Fuchs, H. (2009). Comparison of different image fusion techniques for individual tree crown identification using QuickBird images. International Society for Photogrammetry and Remote Sensing, High-Resolution Earth Imaging for Geospatial Information, 38, 1-4.

[19] Rockinger, O., \& Fechner, T. (1998, April). Pixel-level image fusion: the case of image sequences. In Proc. SPIE (Vol. 3374, pp. 378-388).

[20] Thomas, C., \& Wald, L. (2006, July). Analysis of changes in quality assessment with scale. In Information Fusion, 2006 9th International Conference on (pp. 1-5). IEEE.

[21] Tu, T. M., Huang, P. S., Hung, C. L., \& Chang, C. P. (2004). A fast intensity-hue-saturation fusion technique with spectral adjustment for IKONOS imagery. IEEE Geoscience and Remote sensing letters, 1(4), 309-312.

[22] van der Meer, F. (2006). The effectiveness of spectral similarity measures for the analysis of hyperspectral imagery. International journal of applied earth observation and geoinformation, 8(1), 317.

[23] Wald, L. (2000, January). Quality of high resolution synthesised images: Is there a simple criterion?. In Third conference" Fusion of Earth data: merging point measurements, raster maps and remotely sensed images"(pp. 99-103). SEE/URISCA.

[24] Xu, J., Guan, Z., \& Liu, J. (2008). An improved IHS fusion method for merging multi-spectral and panchromatic images considering sensor spectral response. Int. Arch. Photogramm. Remote Sens. Spat. Inf. Sci, 37, 1169-1174.

[25] Zhang, Y., \& Hong, G. (2005). An IHS and wavelet integrated approach to improve pan-sharpening visual quality of natural colour IKONOS and QuickBird images. Information Fusion, 6(3), 225-234.

[26] Zhang, H. K., Huang, B., Zhang, M., Cao, K., \& Yu, L. (2015). A generalization of spatial and temporal fusion methods for remotely sensed surface parameters. International Journal of Remote Sensing, 36(17), 4411-4445.

[27] Zhang, H. K., \& Huang, B. (2015). A new look at image fusion methods from a Bayesian perspective. Remote Sensing, 7(6), 6828-6861.

[28] Zhou, J., Civco, D. L., \& Silander, J. A. (1998). A wavelet transform method to merge Landsat TM and SPOT panchromatic data. International Journal of Remote Sensing, 19(4), 743-757. 
Farzaneh DadrasJ avan, Farhad Samadzadegan, Fatemeh Fathollahi; Spectral and Spatial Quality Assessment of IHS and Wavelet Based Pan-sharpening Techniques for High Resolution Satellite I magery, Advances in Image and Video Processing, Volume 6 No 2, April (2018); pp: 1-15

Table 1: Specification of wavelet-based methods

\begin{tabular}{|c|c|c|}
\hline $\begin{array}{l}\text { Metho } \\
\text { d }\end{array}$ & $\begin{array}{c}\text { Detail } \\
\text { Coefficients } \\
\end{array}$ & $\begin{array}{l}\text { Approximation } \\
\text { Coefficients }\end{array}$ \\
\hline SW & $\sum_{j=1} W_{\operatorname{Pan}_{j}}$ & LLMS \\
\hline AW & $\sum_{j=1} W_{\operatorname{Pan}_{j}}$ & MS \\
\hline SWI & $\sum_{j=1} W_{\operatorname{Pan}_{j}}$ & LLI \\
\hline AWI & $\sum_{j=1} W_{\operatorname{Pan}_{j}}$ & 1 \\
\hline SWPC & $\sum_{j=1} W_{\mathrm{Pan}_{j}}$ & LLPC1 \\
\hline AWPC & $\sum_{j=1} W_{\operatorname{Pan}_{j}}$ & PC1 \\
\hline AWLP & $\frac{\mathrm{MS}_{\mathrm{i}}}{\frac{1}{3} \sum_{\mathrm{i}=1}^{3} \mathrm{MS}_{\mathrm{i}}} \sum_{\mathrm{j}=1}^{3} \mathrm{~W}_{\mathrm{Pan}}$ & 1 \\
\hline WWI & $\sum_{j=1} W_{\operatorname{Pan}_{j}}$ & $\mathrm{w} 1 \times L L^{\mathrm{Pan}}+\mathrm{w} 2 \times L L^{\mathrm{I}}$ \\
\hline
\end{tabular}

Table 2: Specification of IHS based methods

\begin{tabular}{cc}
\hline Method & Equation \\
\hline Generalized IHS (GIHS) & $(\mathrm{R}+\mathrm{G}+\mathrm{B}+\mathrm{NIR}) / 4$ \\
$\begin{array}{c}\text { Generalized IHS with } \\
\begin{array}{c}\text { Tradeoff Parameter } \\
\text { (GIHS-TP) }\end{array}\end{array}$ & $\mathrm{I}_{\text {new }}=\mathrm{Pan}-\frac{\mathrm{Pan}-\mathrm{I}}{\mathrm{t}}$ \\
\hline $\begin{array}{c}\text { GIHS with Best Tradeoff } \\
\text { Parameter (GIHS-BTP) }\end{array}$ & $\mathrm{F}_{\text {choi }}=\mathrm{I}_{\text {new }}-\mathrm{I}=\frac{\mathrm{t}-1}{\mathrm{t}}(\mathrm{Pan}$ \\
\hline $\begin{array}{c}\text { GIHS with Adaptive } \\
\text { Weights (GIHS-AW) }\end{array}$ & $\mathrm{F}_{\mathrm{i}}=\frac{\mathrm{Pan}}{\mathrm{I}_{\text {new }}}\left(\mathrm{MS}_{\mathrm{i}}+\delta_{\text {choi }}\right)$ \\
\hline $\begin{array}{c}\text { Improved GIHS with } \\
\text { Adaptive Weights } \\
\text { (IGIHS-AW) }\end{array}$ & $\sum_{\mathrm{i}=1}^{\mathrm{n}} \mathrm{w}_{\mathrm{i}} \cdot \mathrm{MS}_{\mathrm{i}}+\mathrm{b}$ \\
\hline $\begin{array}{c}\text { Improved GIHS with } \\
\text { Adaptive Weights } \\
\text { (IGIHS-AW) }\end{array}$ & $\mathrm{I}=\sum_{\mathrm{i}=1}^{\mathrm{n}} \mathrm{w}_{\mathrm{i}} \cdot \mathrm{MS}_{\mathrm{i}}$ \\
\hline
\end{tabular}


Table 3: Fusion quality assessment metrics

\begin{tabular}{|c|c|}
\hline Correlation Coefficients & $\operatorname{corr}=\frac{\sum_{1}^{M} \sum_{1}^{N}(x-\bar{x}) \cdot(y-\bar{y})}{\sqrt{\sum_{1}^{M} \sum_{1}^{N}(x-\bar{x})^{2} \cdot \sum_{1}^{M} \sum_{1}^{N}(y-\bar{y})^{2}}}$ \\
\hline Universal Quality Index & $Q=\frac{\sigma_{x y}}{\sigma_{x} \cdot \sigma_{y}} \cdot \frac{2 \cdot \overline{x y}}{\bar{x}^{2}+\bar{y}^{2}} \cdot \frac{2 \sigma_{x} \cdot \sigma_{y}}{\sigma_{x}^{2}+\sigma_{y}^{2}}$ \\
\hline Spectral Angle Mapper (SAM) & $\cos (\alpha)=\frac{\sum_{i=1}^{N} A_{i} \cdot B_{i}}{\sqrt{\sum_{i=1}^{N} A_{i} \cdot A_{j}} \cdot \sqrt{\sum_{i=1}^{N} B_{i} \cdot B_{j}}}$ \\
\hline ERGAS & $E R G A S=100 \frac{G S D_{P a n}}{G S D_{M S}} \sqrt{\frac{1}{S} \sum_{i=1}^{S}\left[\frac{R M S E\left(B_{i}\right)^{2}}{\bar{x}_{i}^{2}}\right.}$ \\
\hline Spectral Correlation Mapper & $C_{x y}=\frac{1}{\sqrt{\sum(x-\bar{x})^{2} \cdot \sum(y-\bar{y})^{2}}}$ \\
\hline
\end{tabular}

Table 4: Spectral quality metrics calculated for different Wavelet based fusion methods

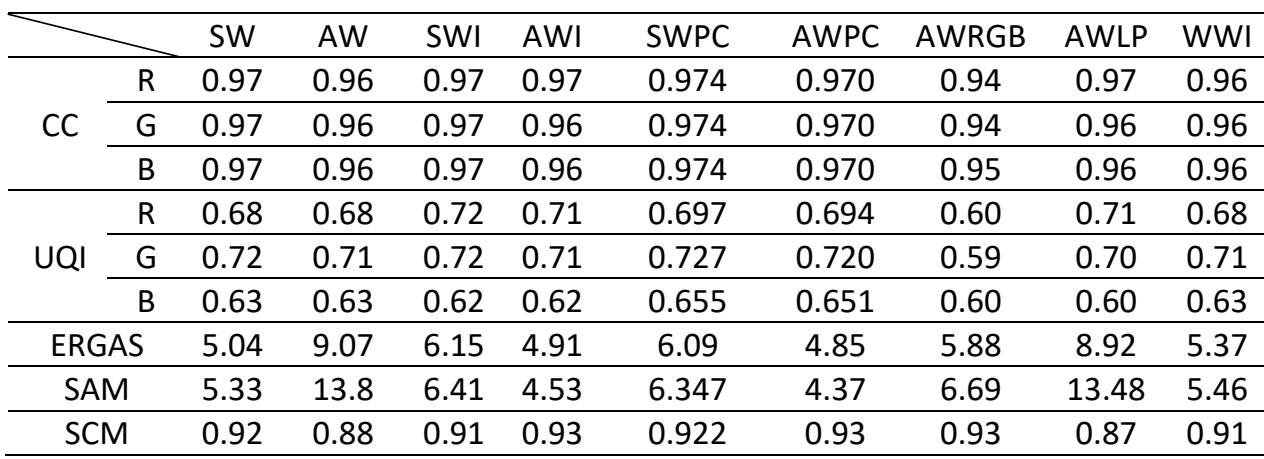

Table 5: Spectral quality metrics calculated for different IHS based fusion methods

\begin{tabular}{|c|c|c|c|c|c|c|c|}
\hline & & IHS & GIHS & $\begin{array}{c}\text { GIHS- } \\
\text { TP }\end{array}$ & $\begin{array}{l}\text { GIHS- } \\
\text { BTP }\end{array}$ & $\begin{array}{l}\text { GIHS- } \\
\text { AW }\end{array}$ & $\begin{array}{c}\text { IGIHS- } \\
\text { AW }\end{array}$ \\
\hline \multirow{3}{*}{$\mathrm{CC}$} & $R$ & 0.86 & 0.94 & 0.96 & 0.94 & 0.96 & 0.97 \\
\hline & G & 0.84 & 0.92 & 0.95 & 0.93 & 0.95 & 0.97 \\
\hline & $B$ & 0.80 & 0.92 & 0.95 & 0.92 & 0.95 & 0.97 \\
\hline \multirow{3}{*}{ UQI } & $R$ & 0.55 & 0.65 & 0.72 & 0.66 & 0.66 & 0.79 \\
\hline & G & 0.53 & 0.64 & 0.72 & 0.65 & 0.64 & 0.80 \\
\hline & B & 0.42 & 0.53 & 0.61 & 0.55 & 0.55 & 0.80 \\
\hline \multicolumn{2}{|c|}{ ERGAS } & 12.0 & 7.95 & 6.36 & 7.69 & 6.37 & 5.28 \\
\hline \multicolumn{2}{|c|}{ SAM } & 16.9 & 5.00 & 3.96 & 3.96 & 4.25 & 0.02 \\
\hline \multicolumn{2}{|c|}{ SCM } & 0.58 & 0.79 & 0.86 & 0.80 & 0.90 & 0.93 \\
\hline
\end{tabular}


Farzaneh Dadrasjavan, Farhad Samadzadegan, Fatemeh Fathollahi; Spectral and Spatial Quality Assessment of IHS and Wavelet Based Pan-sharpening Techniques for High Resolution Satellite Imagery, Advances in I mage and Video Processing, Volume 6 No 2, April (2018); pp: 1-15

Table 6: Spatial quality metrics calculated for different Wavelet based fusion methods.

\begin{tabular}{|c|c|c|c|c|c|c|c|c|c|c|}
\hline \multicolumn{2}{|c|}{$\begin{array}{l}\text { Metrics } \\
\text { Methods }\end{array}$} & SW & AW & SWI & AWI & SWPC & AWPC & AWRGB & AWLP & WWI \\
\hline \multirow{3}{*}{ C } & \multirow{2}{*}{$\begin{array}{l}\mathrm{R} \\
\mathrm{G}\end{array}$} & 0.993 & 0.935 & 0.933 & 0.868 & 0.868 & 0.885 & 0.922 & 0.89 & 0.902 \\
\hline & & 0.941 & 0.943 & 0.933 & 0.932 & 0.933 & 0.944 & 0.931 & 0.95 & 0.958 \\
\hline & $B$ & 0.882 & 0.885 & 0.921 & 0.920 & 0.922 & 0.934 & 0.869 & 0.82 & 0.946 \\
\hline \multirow{3}{*}{ UQI } & $R$ & 0.811 & 0.799 & 0.638 & 0.635 & 0.635 & 0.683 & 0.762 & 0.77 & 0.743 \\
\hline & $\mathrm{G}$ & 0.807 & 0.804 & 0.767 & 0.762 & 0.765 & 0.803 & 0.761 & 0.84 & 0.852 \\
\hline & $B$ & 0.693 & 0.685 & 0.751 & 0.750 & 0.757 & 0.797 & 0.641 & 0.67 & 0.834 \\
\hline \multirow{3}{*}{$\mathrm{sCC}$} & $\mathrm{R}$ & 0.993 & 0.969 & 0.974 & 0.971 & 0.974 & 0.965 & 0.977 & 0.995 & 0.991 \\
\hline & $\mathrm{G}$ & 0.994 & 0.970 & 0.981 & 0.978 & 0.981 & 0.971 & 0.978 & 0.981 & 0.997 \\
\hline & $B$ & 0.989 & 0.966 & 0.978 & 0.976 & 0.978 & 0.970 & 0.973 & 0.972 & 0.994 \\
\hline \multicolumn{2}{|c|}{ ERGASs } & 11.4 & 11.94 & 11.55 & 11.9 & 11.56 & 11.9 & 12.9 & 13 & 10.18 \\
\hline
\end{tabular}

Table 7: Spatial quality metrics calculated for different IHS based fusion methods.

\begin{tabular}{|c|c|c|c|c|c|c|c|}
\hline \multicolumn{2}{|c|}{ Metric } & IHS & GIHS & $\begin{array}{c}\text { GIHS- } \\
\text { TP }\end{array}$ & GIHS-BTP & GIHS-AW & IGIHS-AW \\
\hline \multirow{3}{*}{$\mathrm{CC}$} & $\mathrm{R}$ & 0.97 & 0.94 & 0.92 & 0.94 & 0.85 & 0.85 \\
\hline & $\mathrm{G}$ & 0.99 & 0.98 & 0.97 & 0.98 & 0.92 & 0.92 \\
\hline & $B$ & 0.97 & 0.89 & 0.87 & 0.88 & 0.77 & 0.77 \\
\hline \multirow{3}{*}{ UQI } & $R$ & 0.86 & 0.86 & 0.82 & 0.86 & 0.73 & 0.69 \\
\hline & G & 0.85 & 0.86 & 0.88 & 0.86 & 0.82 & 0.78 \\
\hline & $\mathrm{B}$ & 0.75 & 0.75 & 0.71 & 0.74 & 0.63 & 0.57 \\
\hline \multirow{3}{*}{$\mathrm{sCC}$} & $R$ & 0.99 & 0.98 & 0.98 & 0.98 & 0.98 & 0.95 \\
\hline & $\mathrm{G}$ & 0.99 & 0.99 & 0.98 & 0.99 & 0.98 & 0.96 \\
\hline & B & 0.99 & 0.99 & 0.99 & 0.99 & 0.96 & 0.91 \\
\hline \multicolumn{2}{|c|}{ ERGASs } & 4.6 & 7.5 & 8.4 & 7.7 & 12.0 & 11.9 \\
\hline
\end{tabular}

Table 8: Summarized grades of Wavelet based methods

\begin{tabular}{cccccccccc}
\hline & SW & AW & SWI & AWI & SWPC & AWPC & AWRGB & AWLP & WWI \\
\hline Spectral Quality & 0.61 & 0.63 & 0.66 & 0.56 & 0.73 & 0.64 & 0.21 & 0.63 & 0.49 \\
\hline Spatial Quality & 0.68 & 0.44 & 0.47 & 0.41 & 0.43 & 0.44 & 0.47 & 0.55 & 0.71 \\
\hline Quality & 0.83 & 0.45 & 0.53 & 0.33 & 0.53 & 0.46 & 0.10 & 0.63 & 0.76 \\
\hline
\end{tabular}

Table 9: Summarized grades of IHS based methods

\begin{tabular}{ccccccc}
\hline & IHS & GIHS & GIHS-TP & GIHS-BTP & GIHS-AW & IGIHS-AW \\
\hline Spectral Quality & 0.45 & 0.79 & 0.67 & 0.51 & 0.66 & 0.65 \\
\hline Spatial Quality & 0.82 & 0.79 & 0.75 & 0.79 & 0.59 & 0.42 \\
\hline Quality & 0.50 & 0.96 & 0.73 & 0.55 & 0.52 & 0.29 \\
\hline
\end{tabular}




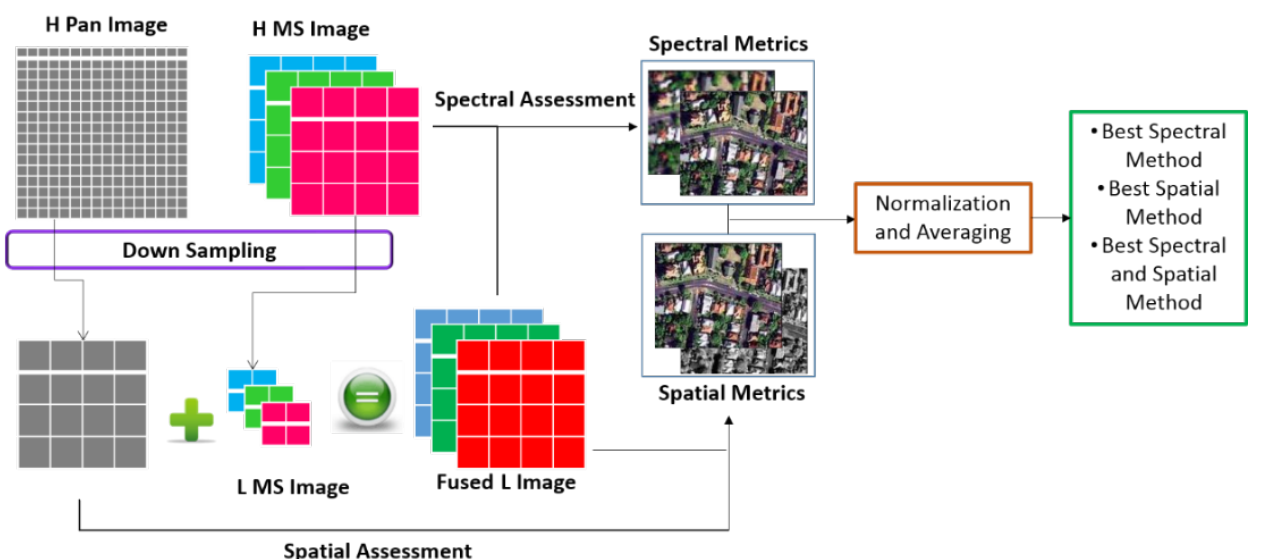

Figure 11: Image Fusion quality assessment strategy

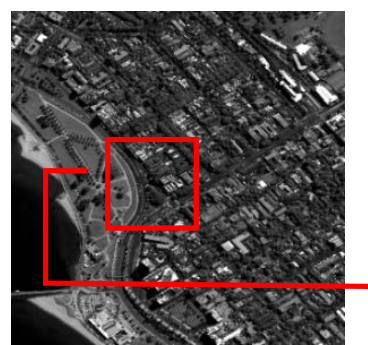

a

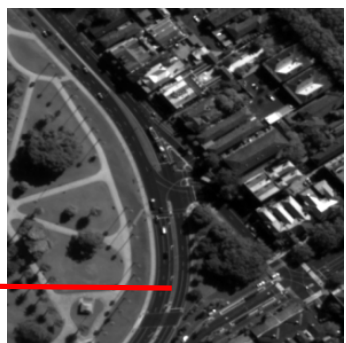

b

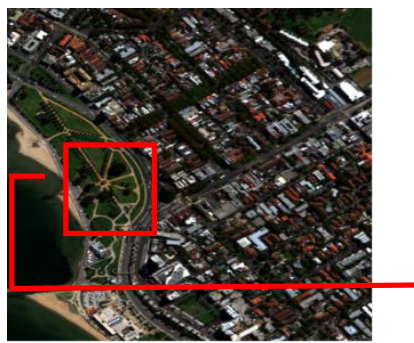

C

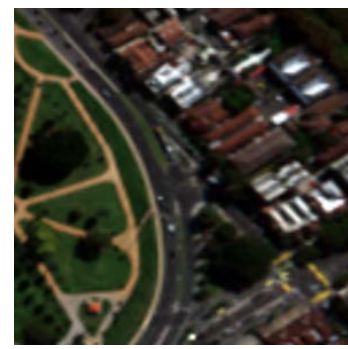

d

Figure 2: Image dataset. a) Original pan image with $0.5 \mathrm{~m}$ resolution. b) Selected part of original pan image (500 $\times 500$ pixels). c) RGB combination of the up-sampled MS image with $2 \mathrm{~m}$ resolution. d) Selected part of the RGB combination of up-sampled MS (500×500 pixels).

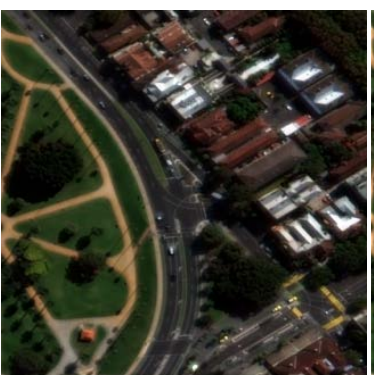

a

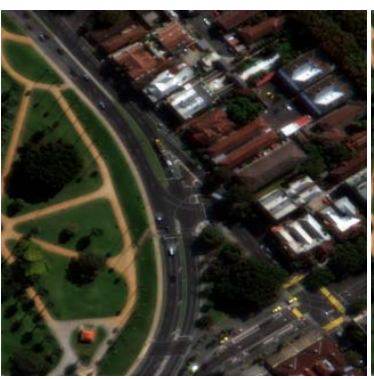

e

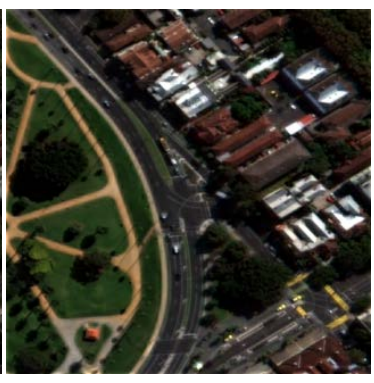

b

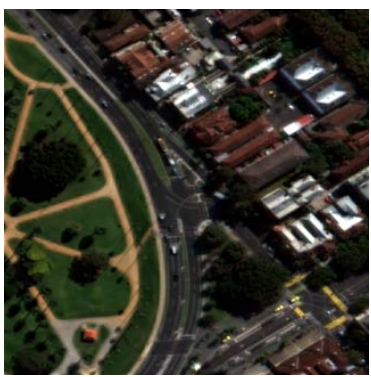

f

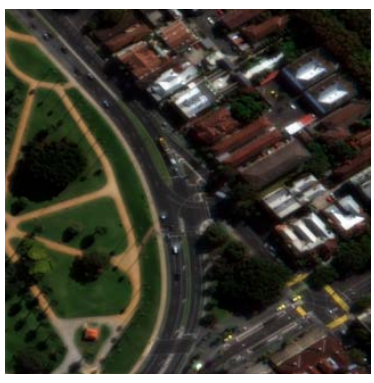

C

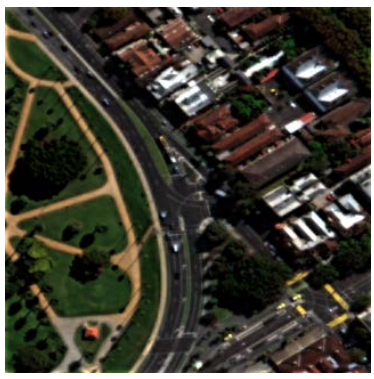

g

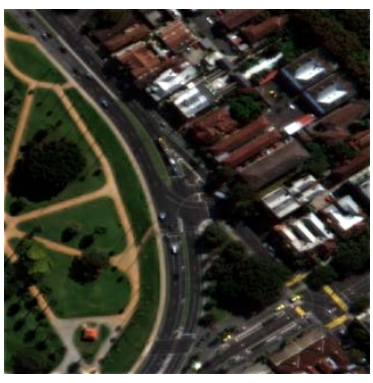

d

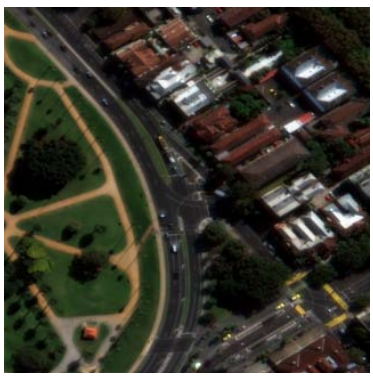

h

Figure 3: Results of the applied wavelet-based pan-sharpening methods. a) SW b) AW c) SWI d) AWI e) SWPC f) AWPC g) AWLP h)WWI 
Farzaneh DadrasJ avan, Farhad Samadzadegan, Fatemeh Fathollahi; Spectral and Spatial Quality Assessment of I HS and Wavelet Based Pan-sharpening Techniques for High Resolution Satellite Imagery, Advances in I mage and Video Processing, Volume 6 No 2, April (2018); pp: 1-15

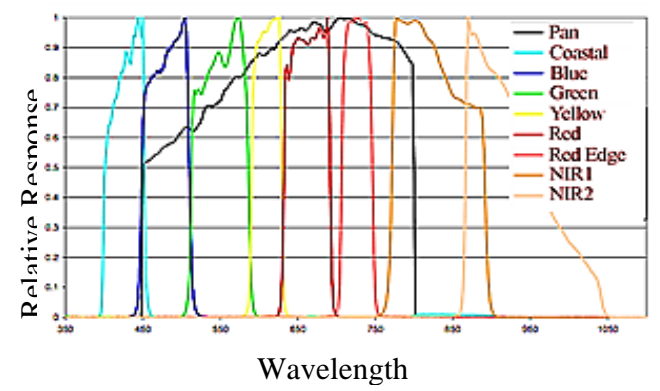

Figure 4: Spectral response of the WV2 sensors

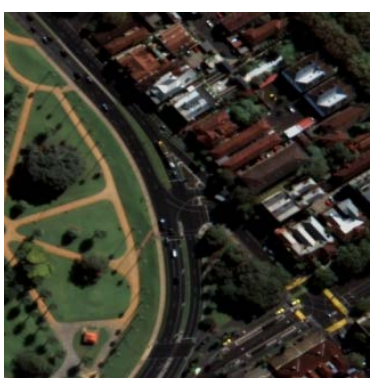

a

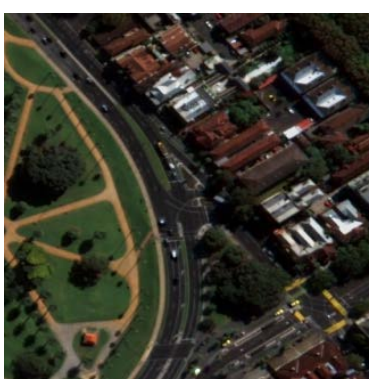

b

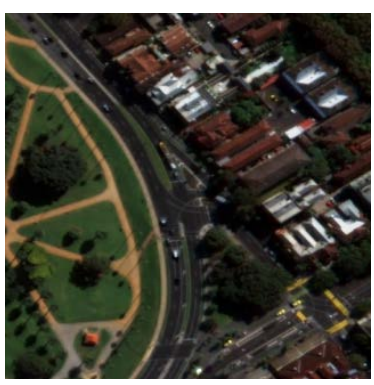

c

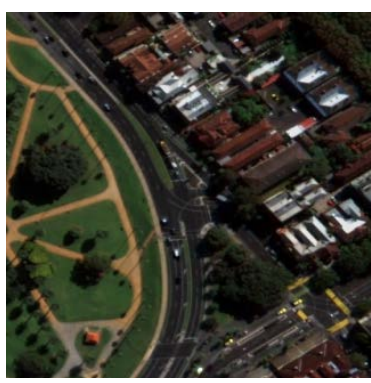

d

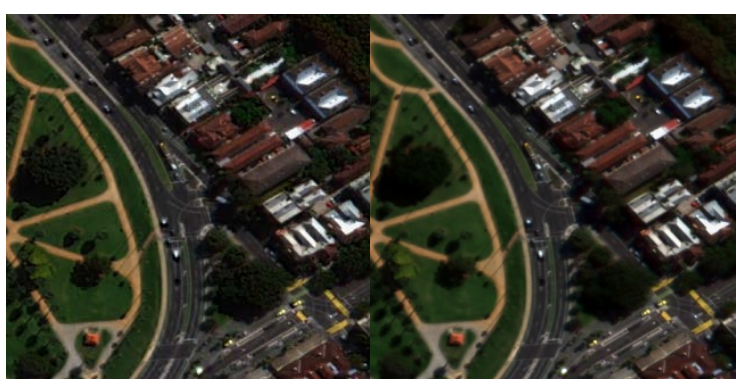

e

$\mathrm{f}$

Figure 5: Results of the IHS-based pan-sharpening methods. a) Traditional IHS b) GIHS c) GIHS-TP d) GIHSBTP e) GIHSAW f) IGIHS-AW
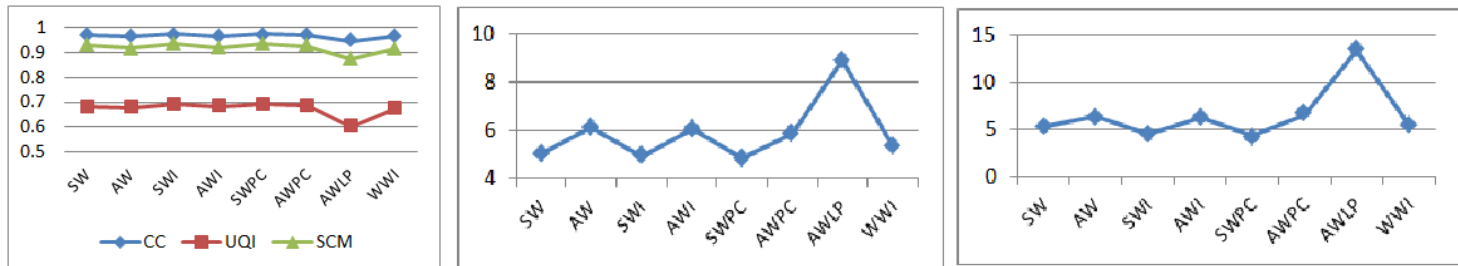

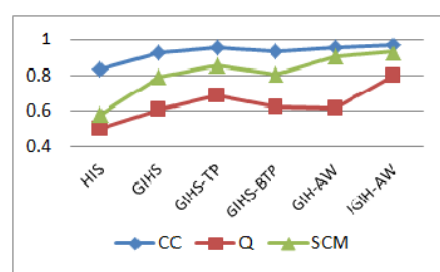

a. CC, Q, SCM

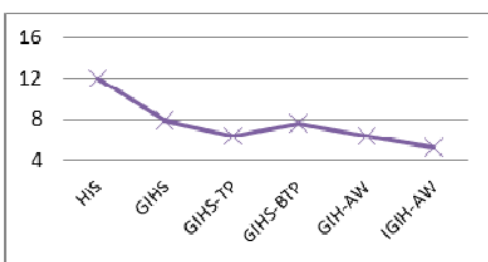

b. ERGAS

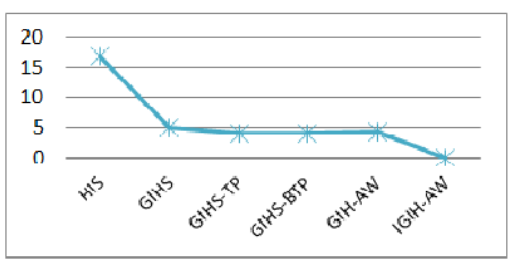

c. SAM

Figure 6: Spectral quality metrics calculated for different methods. above) Wavelet based methods, below) IHS based methods 

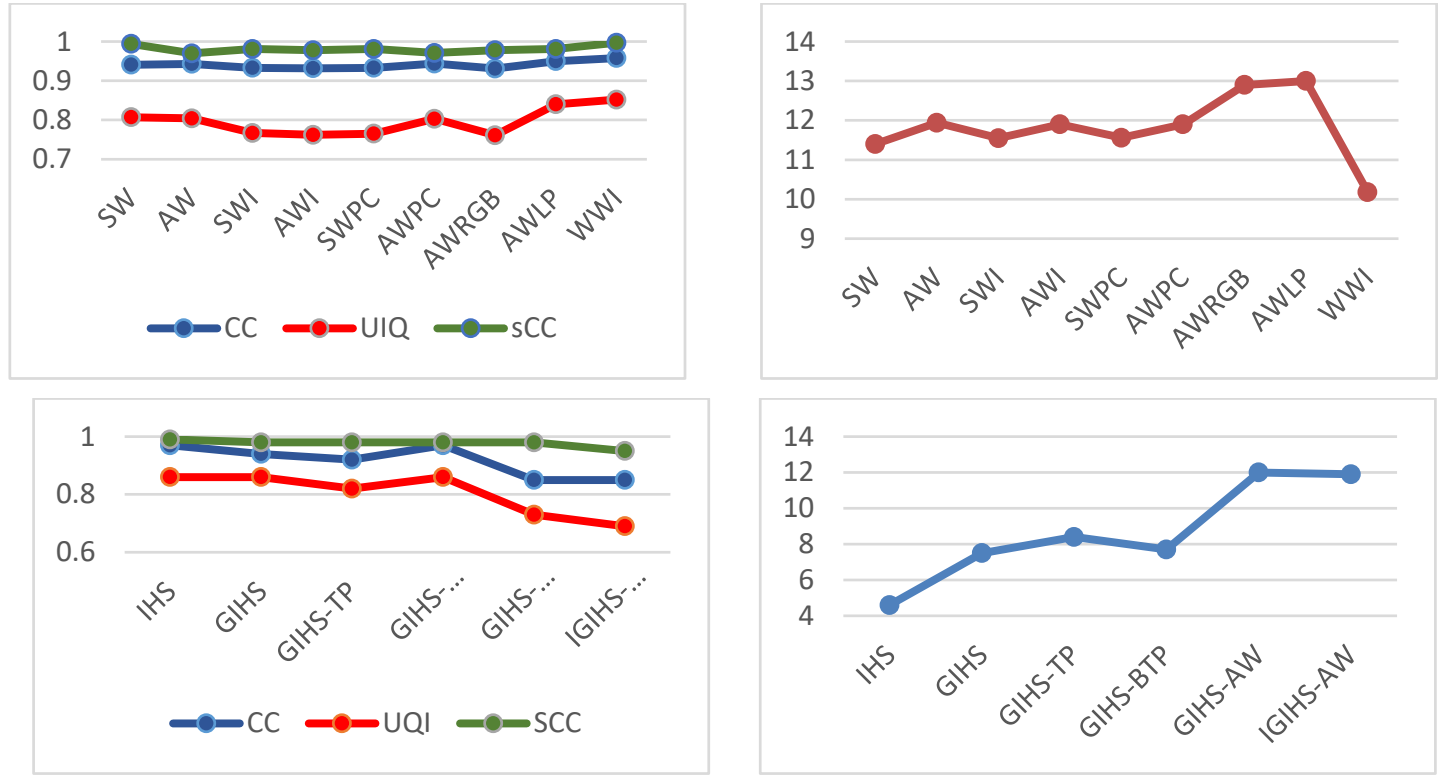

a) CC,UQI and SCC(averaged over three bands of $R, G$ and $B$ )

b) ERGASs

Figure 7: Spatial quality metrics calculated for different methods. above) Wavelet based methods, below) IHS based methods 